\title{
Simulative study of the decentralized control of complex conveyor networks
}

\author{
DIPL.-ING. DIRK LIEKENBROCK \\ FRAUNHOFER-INSTITUT FÜR MATERIALFLUSS UND LOGISTIK IML, DORTMUND
}

\begin{abstract}
Decentralised controls offer advantages for the implementation as well as the operation of controls of steady conveyors. Such concepts are mainly based on RFID. Due to the reduced expense for appliances and software, however, the plant behaviour cannot be determined as accurately as in centrally controlled systems. This article describes a simulation-based method by which the performances of these two control concepts can easily be evaluated in order to determine the suitability of the decentralised concept.
\end{abstract}

\section{Introduction}

Typical controls of general steady conveyors are hierarchically organised. By dividing the system into different levels where each area has its own function the availability and maintenance of the system should be improved and it should be possible to integrate automation components of different suppliers. But when it grows a system becomes more complex and the system behaviour is difficult to plan and master. For this reason, the choice and evaluation of control methods for the master system are a regular part of a simulation-based planning to achieve an efficient system design and to prepare for the implementation of the transport control. The simulation of the material flow may require a large part of the complete project.

Decentralised control methods are an interesting, yet not sufficiently studied alternative. In each area self-operating control hubs are responsible for the control without a master system by cyclically exchanging information about routings and occupation states. Despite easily describable control methods, however, the expenses for the determination of throughput-optimised control parameter in an event-discrete simulation model are high compared to centralised controls.

This article describes an approach towards a comprehensive study of distributed control strategies in complex general steady conveyors which are controlled by decentralised hubs. A material flow model which has been abstracted and extended on the basis of irreducible transport hubs set the basis for the development of an assistant system which is studied and evaluated below.

\section{The problem}

Unlike in a centrally controlled conveyor system the flow rate and the distribution of transport times of a decentrally controlled system can be determined by simulation prior to the realisation. Thanks to their precise representation event-discrete material flow simulators are especially suitable for the simulation of complex conveyor systems. System models can easily be developed with pre-configured model elements and the process is programmed according to the model. This programming includes the routing at branchings and the sequencing of goods flows at junctions. Both program parts may be statical or dynamical. Statical strategies are simple handling rules at junctions or fixed routing rules. Depending on the function the number of such rules is limited. Dynamical strategies furthermore include the recording of the load status in plant components in order to be able to react to fluctuating flow rates.

Dynamical, i.e. load-adaptive strategies may have an important effect on the flow rate and result in a better system behaviour than statical strategies. It is quite difficult, however, to program and carry out the simulation with adaptive strategies because iterative simulation processes are required and the basic strategies have to be adapted to achieve an optimized plant behaviour [Bramel97]. In case of complex models with a large number of model elements this requires extensive adaptations and a long simulation time [Fowler04]. 


\section{Procedure}

In addition to an event-discrete just-in- time modelling conveyor processes can also be represented in a location-discrete way. In this representation the sections of a conveyor system are divided into $i$ segments with a length of $l_{\mathrm{k}}(k \in[1 ; i])$ which may be occupied by a transport object. A conveyor process is the synchronised moving of an object along the segments. Because the segments may be randomly short the representation can be as exact in the location and time area as in an event-discrete simulation. The time base $\mathrm{T}$ of this movement is constant and is carried out synchronously for all segments. The conveyor speed $v_{k}$ in a segment $k$ is

$$
v_{k}=\frac{l_{k}}{T} .
$$

The calculations in a location-discrete model can be reduced considerably by prolonging the segments because this will reduce the number of events. But this abstraction will also make the representation less accurate. The representation accuracy of location-discrete models of steady conveyors for piece goods therefore has to be evaluated in a direct comparison with the eventdiscrete simluation in order to verify its suitability for practical use.

This study is based on the model of the irreducible transport hubs [Großeschallau84] (fig. 1) which differentiates between the three elements track, switch and junction in order to simulate the conveyor elements.
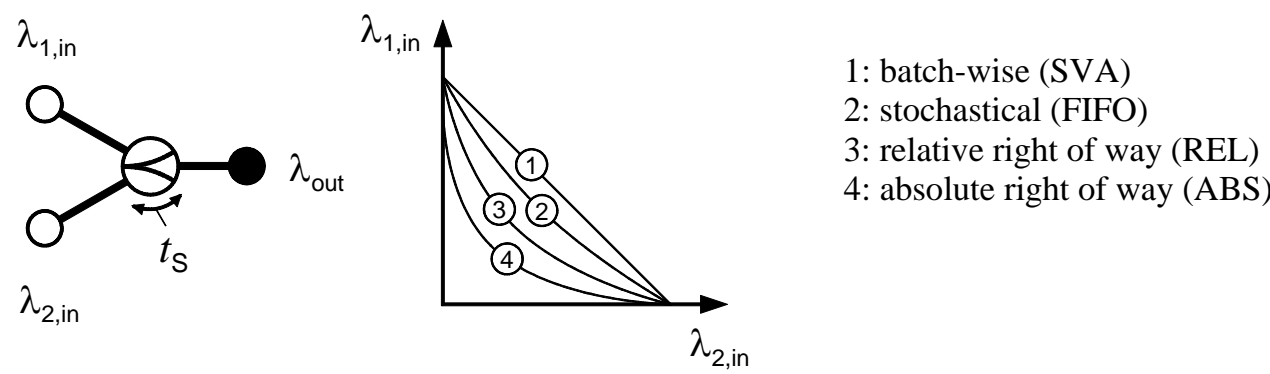

Fig. 1: Variables at a junction and effect of the strategy on the flow rate

Owing to the location-discrete modelling the time set-off for the given conveying speed is only small at tracks and switches compared to continuous event-discretely modelled conveying processes. At junctions, however, the predetermined handling strategies may lead to a variation of lead and waiting times for piece goods (transport objects) before the elements. This can have a decisive influence on the distribution of these time shares within a more complexe model consisting of many hubs. The central aspect of this study therefore is the junction element.

The model consists of a junction with two input tracks where goods sequences with randomly distributed intermediate arrival times are dispatched and which are handled by the junction element according to the strategy. For each transport object the time when it enters and leaves the model and thus the lead and waiting times can be determined individually (fig. 2).
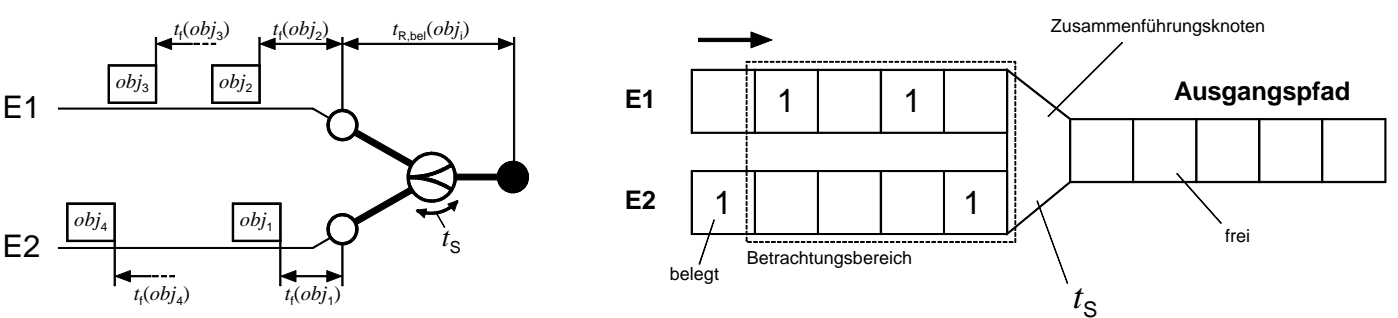

Fig. 2: Comparison of a continuous and a location-discrete model 
The simulated container conveyor system holds containers of equal length. For the locationdiscrete model the segment length is set to the length of one container.

The input sequences at the paths E1 and E2 are set as continuous, randomly distributed input times with an average intermediate arrival time $\lambda$ m between two objects. For the location-discrete model the input times have been converted into integral values on the time basis T. Each of the generated input sequences is simulated according to four strategies (fig. 1) for both cases. The result are the total lead time TLT as well as the sum of the path-related waiting times WT1 and WT2.

\section{Results}

The $\mathrm{x}$-coordinate in figure 3 shows the accumulated distribution of the relative deviations between the location-discrete and the continuous simulation of these three variables. In the location-discrete simulation the chance that two containers arrive at the junction simultaneously sinks in line with a sinking intermediate arrival time. Stochastic and batch-wise handling can only be carried out at random. For this reason the output sequences of both simulations may differ by pairs. The bit error rate as indicator for containers which have been confused at the junction by pairs indicates the frequency of these confusions during a predetermined input sequence.
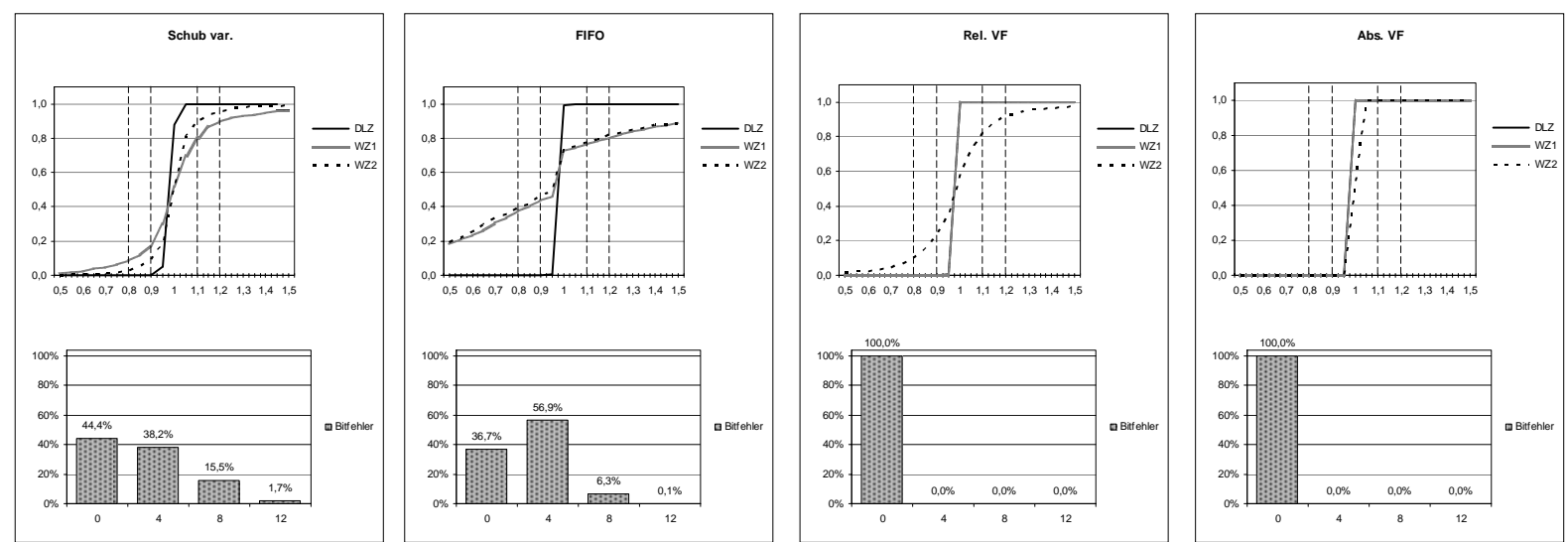

Parameter: $2 \times 20$ objects, $\lambda_{\mathrm{m}}=2 \mathrm{~s}, t_{\mathrm{S}}=1$

Fig. 3: Relative deviations and bit errors of TLT and WT in the location-discrete simulation (kum.)

Because of the small number of confusions in the location-discrete simulation the lead and waiting times for single objects are rather short at a junction compared to the event-discrete simulation. In the studied cases the maximum number of bit errors was twelve, i.e. six transport objects each on both input paths were handled in the wrong order and thus constituted a TLT and WT error of \pm 1 segment length. This has a decisive effect on the presented frequency distribution: in the ideal case the time in the location-discrete and continuous simulation always remains 1 . This, however, is only the case for the absolute right of way.

\section{Further projects}

The results clearly show possible deviations at different input sequences, intermediate arrival times and handling strategies at junctions. Furthermore, first comparing simulations of networks with several switches and junctions show deviations of less than $10 \%$ between object-related lead or waiting times and event-discrete simulations. Since the lead times of the handling strategies "Batch-wise handling" and "Absolute right of way" at only one junction already differ by $100 \%$ and the distribution of waiting times may differ even more this method can principally be applied.

Exemplatory studies of segments show that these methods can also be used in large networks. The study describes a model with 406 adequate segments and 22 freely parameterisable junctions 
as strategic elements. The calculation time required for the simulation of conveying processes is proportionate to the number of model segments, the necessary runtime can be converted linearly into other models by means of the number of segments. In order to illustrate the runtime behaviour input sequences with a different number of objects were despatched along the transport relation into a source of the model and the simulation time was recorded (fig. 4):

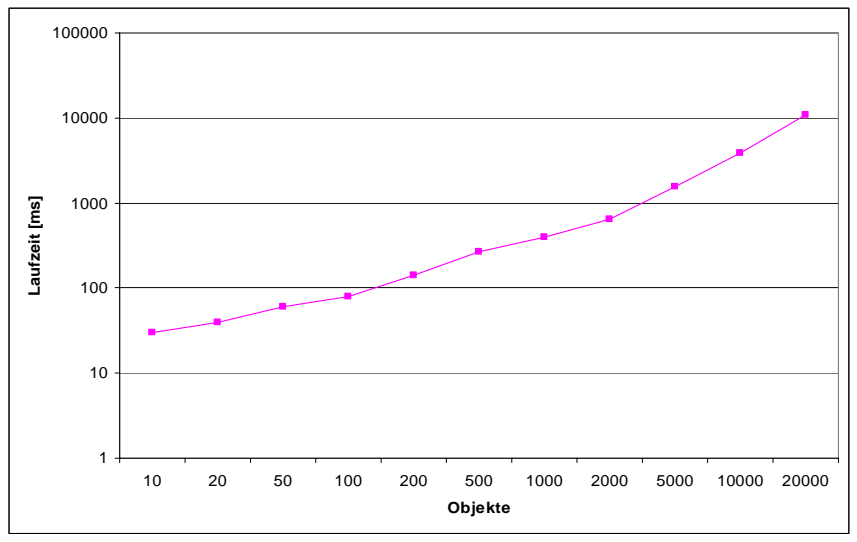

INTEL Pentium M; $1,8 \mathrm{GHz}, 1 \mathrm{~GB}$

Fig. 4: Calculation time according to the number of objects (continuous despatch)

In the studied case the event-discrete simulation can thus be used to simulate all statical combination of strategies at the junctions in a finite runtime. Including load states on tracks this allows for the study of dynamical, load-adaptive strategies for centralised control systems. It is planned to extend the assistant systems by the simulation of an autonomous object behaviour where control decisions are made locally at the switches and junctions. Thus, centralised and decentralised control configurations with identical input sequences can directly be compared and evaluated.

\section{Literature}

[Bramel97] Bramel, Julien; Simchi-Levi, Daniel: The logic of logistics - Theory, algorithms, and applications for logistics. (Springer series in operations research). Springer Verlag, 1997

[Fowler04] Fowler, John W.; Rose, Oliver: Grand Challenges in Modeling and Simulation of Complex Manufacturing Systems, SIMULATION, Vol. 80, No. 9, Sept. 2004, pp 469- 476

[Großeschallau84] Großeschallau, Werner; Jünemann, Reinhard (Ed.): Materialflußrechnung, Springer Verlag, 1984 